\title{
MODELAGEM FRACIONÁRIA: O CASO DAS ONDAS ELETROMAGNÉTICAS
}

\author{
José Humberto de Souza Prates ${ }^{1}$; Davidson Martins Moreira² \\ ${ }^{1}$ Campus Integrado de Manufatura e Tecnologia SENAI CIMATEC, Salvador/BA; \\ hprates2005@hotmail.com \\ ${ }^{2}$ Campus Integrado de Manufatura e Tecnologia SENAI CIMATEC, Salvador/BA; \\ davidson.moreira@gmail.com
}

Resumo: Embora invisíveis para nós, as ondas eletromagnéticas estão presentes em nosso cotidiano, sendo útil em diversas áreas. Na geofísica por exemplo aparece como muita frequência nos métodos eletromagnéticos. As ondas eletromagnéticas surgem das equações de Maxwell, que são equações lineares, e dão origem a equações diferenciais, também lineares. Estas podem ser modeladas, lançando mão das equações diferenciais fracionárias, levando a uma interpretação mais acurada do fenômeno. Neste trabalho será utilizada uma nova metodologia para resolução de equações diferenciais fracionárias, proposta em Khalil(2014). Também neste trabalho uma correlação entre os parâmetros do meio e o exponente fracionário será proposta, trazendo assim uma contribuição para a busca do entendimento da física do expoente fracionário.

Palavras-Chave: ondas eletromagnéticas planas; geofísica; equações diferenciais fracionárias; derivadas conformáveis.

\section{FRACTIONAL MODELING: THE CASE OF ELECTROMAGNETIC WAVES}

\begin{abstract}
O Abstract: Although invisible to us, electromagnetic waves are present in our daily lives, being useful in many areas. In geophysics, for example, it appears as often in electromagnetic methods. Electromagnetic waves arise from Maxwell's equations, which are linear equations, and give rise to differential equations, also linear. These can be modeled using fractional differential equations, leading to a more accurate interpretation of the phenomenon. In this work we will use a new methodology for solving fractional differential equations, proposed in Khalil (2014). In this work a correlation between the middle parameters and the fractional exponent will be proposed, thus contributing to the search for understanding the physics of the fractional exponent.
\end{abstract}

Keywords: flat electromagnetic waves; geophysics; fractional differential equations; conforming derivatives. 


\section{INTRODUÇÃO}

Alguns estudos sobre os métodos eletromagnéticos deduzem expressões para o campo magnético através das equações de Maxwell [1],[2] e [9]. As equações resultantes são resolvidas utilizando métodos clássicos de soluções de equações diferenciais, e sempre utilizando a modelagem de ordem inteira. Além disto, estudos envolvendo ondas eletromagnéticas também se utilizam de métodos clássicos e soluções numéricas [5], [6] das equações diferenciais. Neste trabalho, será objeto de análise a propagação de uma onda eletromagnética em um meio utilizando uma nova metodologia para resolução de equações diferenciais fracionárias, o qual reduz o custo computacional e, além disso, obtém-se um resultado mais estável e preciso [7]. A modelagem fracionária em equações diferenciais está em crescimento, no que diz respeito a sua utilização. Historicamente, considera-se como a primeira aplicação do cálculo fracionário a solução do problema da Curva Tautocrônica, proposto por Niels Henrik Abel $_{2}$ em 1820, e trabalhado por Dirichlet ${ }_{2}$ em 1840. Dentre outras aplicações das derivadas fracionárias, existem as seguintes utilizações: no estudo de materiais com memória, fenômenos de difusão, epidemiologia e vibrações mecânicas.

\section{METODOLOGIA}

A escolha das ondas eletromagnéticas como objeto de estudo se deve ao fato de que estas, estão presentes em nosso cotidiano, sendo útil em diversas áreas. Elas podem ser percebidas permeando as teorias da medicina, geofísica, geologia, física, dentre outras. Na geofísica por exemplo aparece como muita frequência nos métodos eletromagnéticos, onde é utilizado campos magnéticos, potenciais elétricos e ondas eletromagnéticas. As ondas eletromagnéticas surgem das equações de Maxwell, que são equações lineares, e dão origem a equações diferenciais também lineares, que serão o foco de nosso trabalho.

Na solução da equação da onda eletromagnética fracionária foi utilizado um método que se baseia numa redefinição da derivada fracionária [7]. Assim, sendo $\alpha \in(n, n+1]$ , e $f$ sendo uma função $n$-diferenciável de $t$, onde $t>0$, então a derivada fracionária conformável de $f$ de ordem $\alpha$ é definida como:

$$
T_{\alpha}(f)(t)=\lim _{\varepsilon \rightarrow 0} \frac{f^{([\alpha]-1)}\left(t+\varepsilon t^{([\alpha]-\alpha)}\right)-f^{([\alpha]-1)}(t)}{\varepsilon}=t^{([\alpha]-\alpha)} f^{[\alpha]}(t)
$$

onde $[\alpha]$ é o menor inteiro maior ou igual a $\alpha$. Tomando-se esta nova definição podese solucionar a equação de onda de uma forma mais simples e com dimensão correta, resultando,

$$
E(z, t)=E_{m}\left[\cos (w t) E_{\alpha}\left(\beta^{\alpha} z^{\alpha}\right)+\operatorname{sen}(w t) E_{\alpha, 2}\left(-\beta^{\alpha} z^{\alpha}\right)\right]
$$

onde $w=2 \pi f$ (frequencia angular), $E_{\alpha}\left(\beta^{\alpha} z^{\alpha}\right)$ e $E_{\alpha, 2}\left(-\beta^{\alpha} z^{\alpha}\right)$ são as funções de MittagLeffler de um e dois parâmetros [4], respectivamente. 


\section{RESULTADOS E DISCUSSÃO}

Por conveniência se faz $w=\beta=1$, e $\alpha=1,9$ temos:

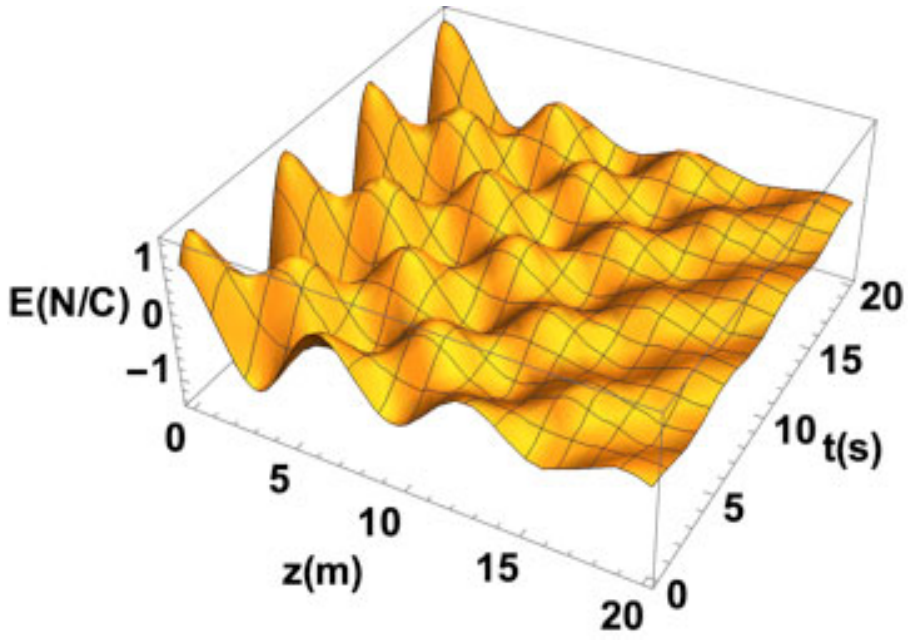

Figura 1 - Gráfico Campo x z x t com $\alpha=1,9$

Na figura 1 com $\alpha=1,9$, observa-se um decaimento na amplitude, lembrando as perdas de energia em meios com condutividade diferente de zero. Para ressaltar esta observação, foi plotado o gráfico campo elétrico $(\mathrm{H}) \times$ posição $(\mathrm{z})$ com $0 \alpha$ variando de 1,7 até 2,0. Nestes gráficos foi fixado o tempo igual ao período. Assim a equação do campo elétrico se reduz a:

$$
E_{(z)}=E_{m} E_{\alpha}\left(\beta^{\alpha} z^{\alpha}\right)
$$

Fazendo por simplicidade $\beta=E_{m}=1$. Pode-se sem perda de generalidade fazer $\beta=1$ , pois estamos no espaço livre onde com certeza este parâmetro é uma constante. Logo:

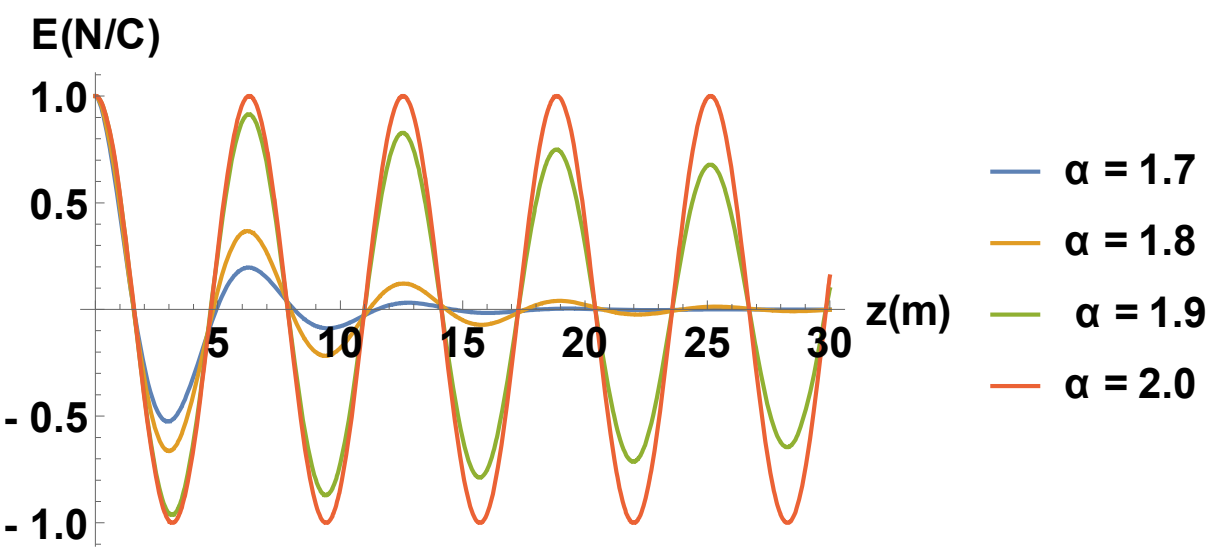

Figura 2: Gráfico Campo x z para $\alpha=1 \cdot 7,1.8,1.9,2.0$

A medida que os campos elétricos e magnéticos se propagam em um meio condutor a sua amplitude é atenuada por um fator $e^{-\psi z}$.[8] Aqui será investigado o formato da envoltória do gráfico campo elétrico $\mathrm{x}$ posição $(\mathrm{z})$, para o caso da modelagem fracionária. a modulação da amplitude apresentada pela maioria dos livros-texto é, na realidade, a envoltória da curva de oscilação, que no caso limite de amortecimentos 
muito pequenos tendem aos mesmos valores numéricos. [4] Aqui neste trabalho será utilizada esta aproximação.

A diferença entre estas duas curvas está apresentada na figura 4.

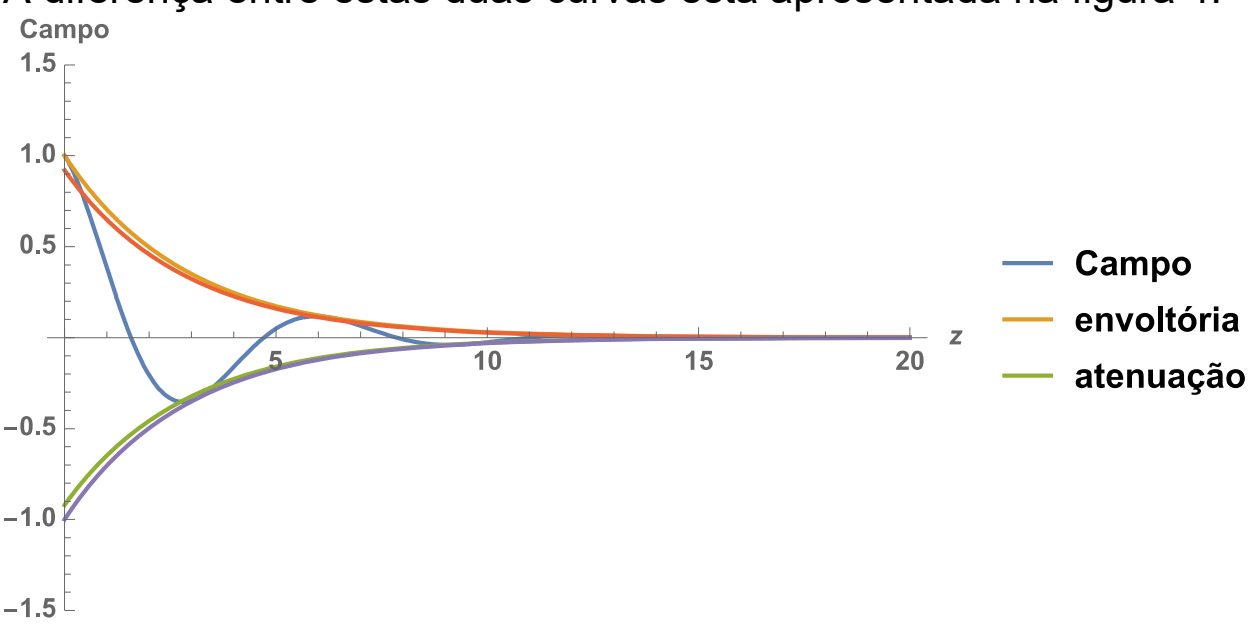

Figura 3 - Gráfico Campo x z apresentando a envoltória e a atenuação

Com esta aproximação foi determinada a equação da envoltória, pegando os pontos de máximo do campo e plotando estes em um gráfico. Após uma análise do formato da curva, ficou evidente que em todos os casos analisados a melhor curva a ser ajustada era a exponencial. Nas figuras 4, 5, 6 e 7 estão exibidas algumas curvas encontradas. Então uma regressão exponencial foi efetivada em cada caso estudado, obtendo assim todas as funções.

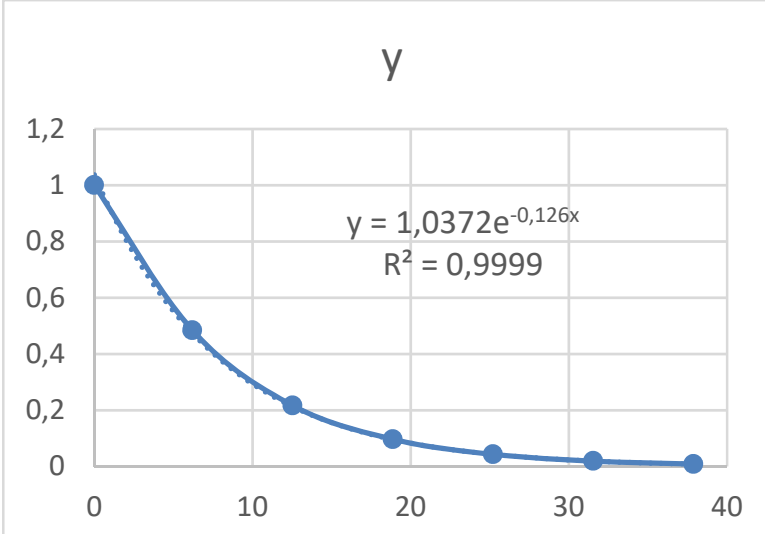

Figura $4-\alpha=1.85$

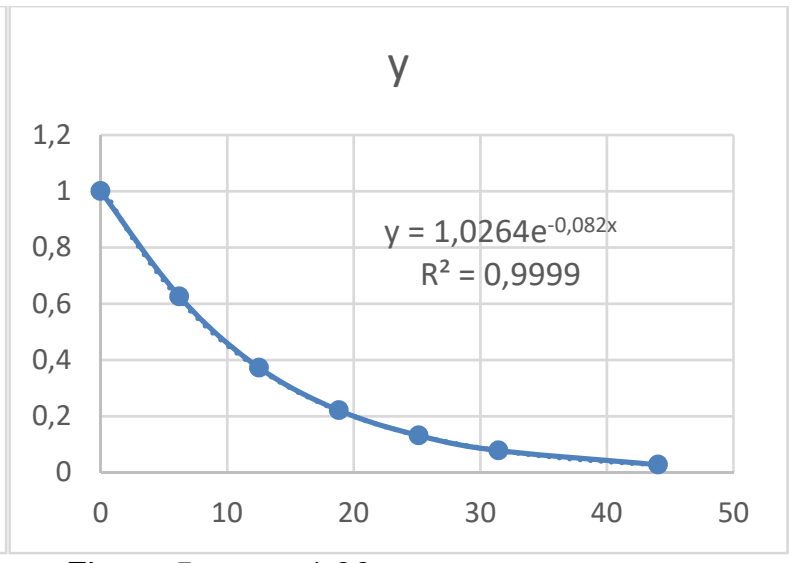

Figura $5-\alpha=1.90$

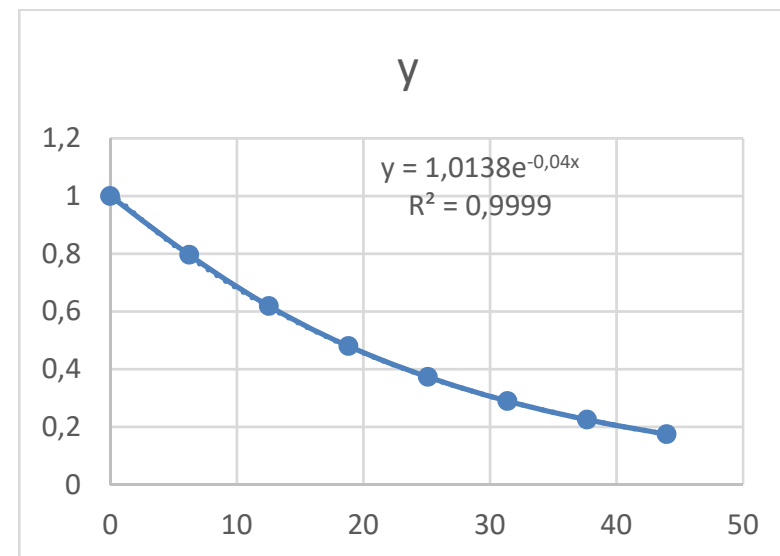

Figura $6-\alpha=1.95$

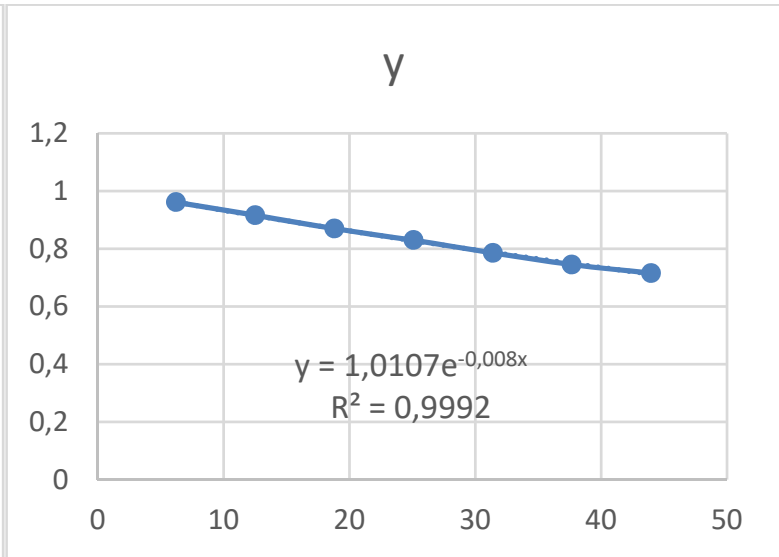

Figura $7-\alpha=1.99$ 
Pode-se observar que todos os coeficientes de determinação das regressões são bem altos(próximos de 1), mostrando assim que todas as curvas obtidas pelas regressões são bem consistentes. Uma outra observação interessante é que quando o $\alpha$ se aproxima de 2, a exponencial se aproxima de uma reta horizontal. O que era de se esperar pois quando o $\alpha$ é 2 é retomada a função de onda sem perdas, onde a envoltória deve ser uma reta constante. Estas constatações retomam a ideia anterior em que a envoltória deve mesmo ter o formato $e^{-\psi z}$. O $\psi$ está evidentemente relacionado com os parâmetros do meio. Parâmetros estes que provocam a atenuação da onda. Uma interessante relação entre $\psi$ e $\alpha$ é encontrada plotando-se o gráfico de dispersão entre estas grandezas. A tabela 1 é obtida após todas as exponenciais entre $\alpha=1.80$ e 1.99 serem conhecidas. Após a plotagem do gráfico observa-se uma forte correlação linear entre $\alpha$ e $\psi$. Possibilitado a regressão linear entre a variável independente $\alpha$ e a dependente $\psi$. Assim chegamos então a relação matemática:

$$
\psi=-0,8657 \alpha+1,7282
$$

\begin{tabular}{|ll|}
\hline$\alpha(\mathrm{x})$ & $\psi(\mathrm{y})$ \\
\hline 1,8 & 0,172 \\
\hline 1,81 & 0,164 \\
1,82 & 0,154 \\
\hline 1,83 & 0,144 \\
\hline 1,84 & 0,135 \\
\hline 1,85 & 0,126 \\
\hline 1,86 & 0,117 \\
\hline 1,87 & 0,108 \\
\hline 1,88 & 0,099 \\
\hline 1,89 & 0,091 \\
\hline 1,9 & 0,082 \\
\hline 1,91 & 0,073 \\
\hline 1,92 & 0,065 \\
\hline 1,93 & 0,056 \\
\hline 1,94 & 0,048 \\
\hline 1,95 & 0,04 \\
\hline 1,96 & 0,032 \\
\hline 1,97 & 0,024 \\
\hline 1,98 & 0,016 \\
\hline 1,99 & 0,008 \\
\hline
\end{tabular}

Figura 8 - Tabela 1

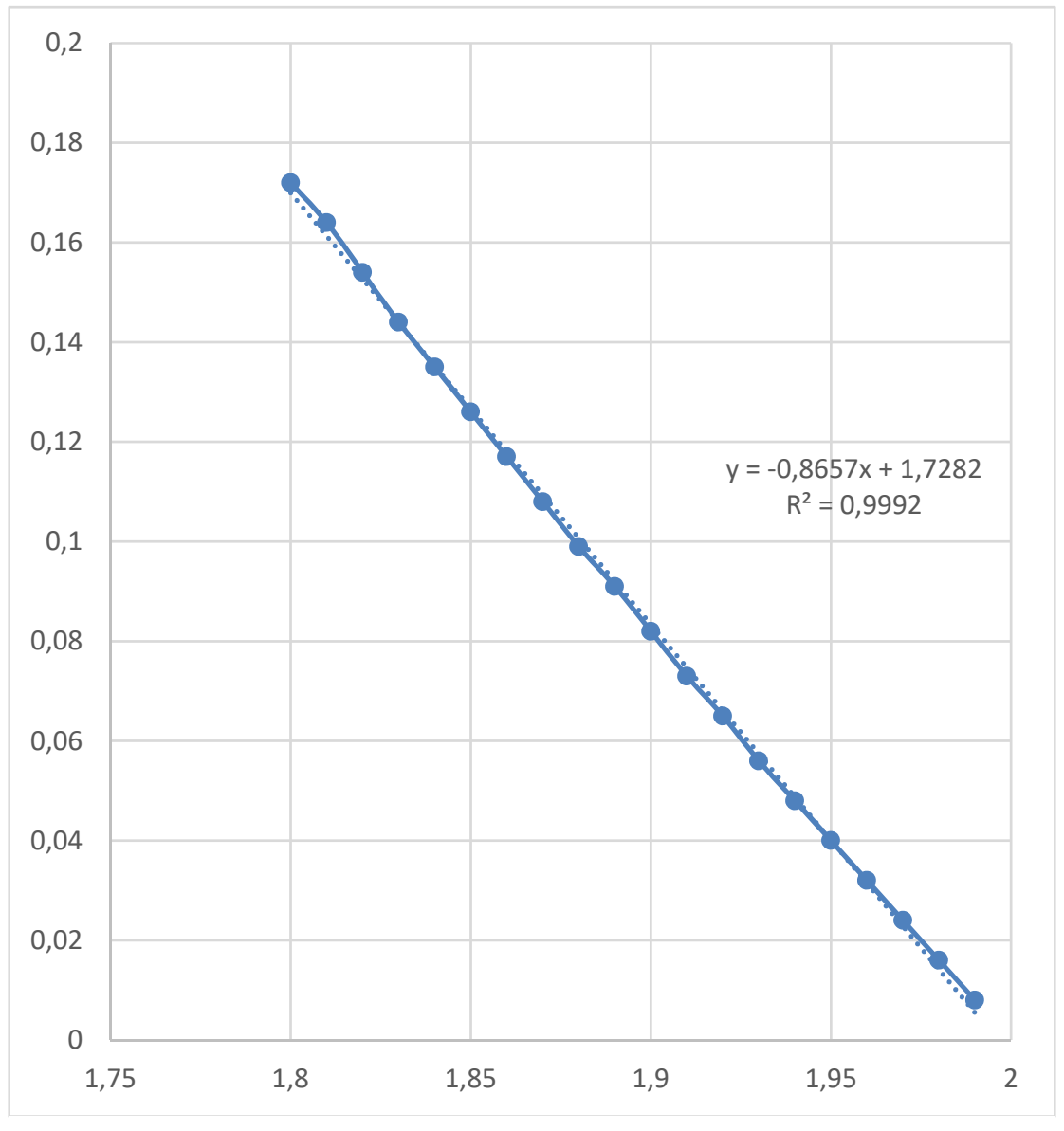

Figura 8 - Gráfico $\alpha(\mathrm{x})$ x parâmetros do meio(y)

Observa-se que existe uma relação linear com o coeficiente de determinação de 0,9992 . Este valor nos trás a certeza desta relação. Pode-se também observar que se extrapolarmos a reta para $\mathrm{y}=\psi=0$, iremos obter $\mathrm{x}=\alpha=2$. Este resultado bate 
claramente com o que era esperado, pois quando temos $\alpha=2$, retomamos a propagação no espaço livre sem perdas, logo $\psi$ tem que ser 0 .

Pode-se, para ter uma solução mais geral, procurar também uma relação entre o $\beta$ (número de onda) e o $\psi$ que representa o expoente da exponencial responsável pelo amortecimento. Será de forma aleatória escolhido $\alpha=1.95$.

\section{CONCLUSÃO}

Os resultados mostram claramente que ao variar o parâmetro fracionário $\alpha$ resgata-se as soluções do amortecimento das ondas em um meio material. Especificamente, quando $\alpha$ é igual a 2 é obtida a solução da propagação da onda no espaço livre. Além disto, uma outra observação interessante é que, quando $\alpha$ se aproxima de 2, a exponencial se aproxima de uma reta horizontal. Isto era esperado, pois quando $\alpha$ é 2 tem-se retomada a função de onda sem perdas, onde a envoltória deve ser uma reta constante. Estas constatações retomam a ideia anterior em que a envoltória deve mesmo ter o formato de $e^{-\psi z}$, onde a constante $\psi$ está evidentemente relacionada com os parâmetros do meio, os quais provocam a atenuação da onda. Finalmente, é importante salientar que o trabalho apresentou uma solução mais geral da equação da onda eletromagnética, aumentando as possibilidades de interpretação do fenômeno.

\section{REFERÊNCIAS}

${ }^{1}$ Chaladgarn, T. and Yooyuanyong, S., 2013. Mathematical model of magnetometric resistivity sounding for a conductive host with a bulge overburden. Applied Mathematical Sciences 7(7), 335-348.

${ }^{2}$ Chen, J. and Oldenburg, D.W., 2004. magnetic and electric fields of direct currents in a layered earth. Exploration geophysics 35, 157-163.

${ }^{3}$ De castro, A.S., 1991. Uma nota de esclarecimento sobre o oscilador harmônico amortecido. Caderno brasileiro de ensino de física 8(2), 137-143.

${ }^{4}$ Mittag-Leffler, G.M., 1903. Sur la nouvelle fonction e_(x). C. R. Acad. Sci. Paris 137, 554-558.

${ }^{5}$ Bulnes, J., Peche, L.A. and Travassos, J.M., 2010. Comment on "frequency-domain green's functions for radar waves in heterogeneous $2.5 \mathrm{~d}$ media". Geophysics, 75(2), $\mathrm{x} 5-\mathrm{x} 5$.

${ }^{6}$ Ellefsen, K.J., Abraham, J.D., Wright, D.I. and Mazzella, A.D., 2004. Numerical study of electromagnetic waves generated by a prototype dielectric logging tool. Geophysics 69(1), 64-77.

${ }^{7}$ Khalil, R., 2014. A new definition of fractional derivative. Journal of Computational and Applied Mathematics 264, 65-70. 
${ }^{8}$ Reitz, J.R., Milford, F.J. and Christy, R.W., 1982. Fundamentos da teoria eletromagnética, Rio de Janeiro. Editora Campus, terceira edição.

${ }^{9}$ Sripanya, W., 2011. Mathematical solutions of electric potential and magnetic field response from heterogeneous media. Tese de Doutorado, Silpakorn University. 